\title{
SHORT DeSign Projects For AN INTRODUCTORY THERMOfLUIDS ENGINEERING COURSE
}

\author{
Michele Hastie* and Jan Haelssig \\ Department of Process Engineering and Applied Science \\ Dalhousie University, Halifax, NS B3H 4R2 \\ *E-mail: michele.hastie@dal.ca
}

\begin{abstract}
The Faculty of Engineering at Dalhousie University offers a common introductory course that covers the basic principles of thermodynamics and fluid mechanics in a unified manner. This introductory course is a mandatory part of the curriculum for all engineering programs offered at Dalhousie. In this course, students are required to perform six laboratory experiments, and since 2012 students have also completed short, four-week design projects.

The short design project helps students to acquire more of the graduate attributes defined by the Canadian Engineering Accreditation Board (CEAB), including design, communication, and team work skills. They also provide students with a well-deserved break from purely theoretical work in lectures and tutorials, and a chance to develop some hands-on abilities.

This paper describes the lessons learned from the last three design projects, which were focused on modifications to a Ranque-Hilsch vortex tube, design of a pop-pop boat, and design of a double pipe heat exchanger. The primary challenges have been the limited engineering design experience possessed by students in their third semester of studies, the heavy workload that second-year engineering students already have, and the relatively large class size. Even though there are clear challenges related to integrating a design project into a large second-year class, the results seem to indicate that these design projects provide a positive learning experience for the students.
\end{abstract}

Keywords: Thermofluids Engineering, Course-integrated Design Projects, Double Pipe Heat Exchanger, Pop-pop Boat, Ranque-Hilsch Vortex Tube.

\section{INTRODUCTION}

Introductory thermodynamics and fluid mechanics courses are a normal part of core engineering curricula at a large fraction of engineering schools across Canada. At Dalhousie University, after an extensive review of the curriculum of the core engineering program, it was decided to offer a common introductory course that covers the basic principles of thermodynamics and fluid mechanics in a unified manner. This introductory course is part of the curriculum for all engineering programs offered at Dalhousie (chemical, civil, electrical, environmental, industrial, materials, mechanical, and mineral resource engineering). The course was first offered in the fall semester of 2011 and covers a wide range of topics, including fluid statics, thermodynamic properties of fluids, applications of the first and second law to closed and open systems, and engineering applications of the Bernoulli equation. Additionally, students perform six laboratory experiments and, since 2012, a short, four-week design project. The last three design projects were focused on modifications to a Ranque-Hilsch vortex tube, design of a pop-pop boat, and design of a double pipe heat exchanger.

The laboratory exercises, and the design project in particular, help students to develop more of the graduate attributes defined by the CEAB. These specific attributes are design, communication and team work skills. The additional advantage of incorporating small design projects into several courses early in the curriculum is that this creates more opportunities to track the development of the attributes throughout the degree. This feedback inherently provides more opportunities to improve the curriculum.

There is evidence that most engineers are active learners, meaning that they process information through active experimentation rather than reflective observation [1]. For a core course that largely consists of passive learning through lectures, incorporating a short design project can help reach out to students who are active learners, which may be the majority of the class. Experiments and design projects also allow students to visualize the physical concepts that they are studying, which generally helps to build a deeper foundational understanding.

Other benefits of short design projects are that they expose students to basic hand tools and construction 
materials and introduce students to open-ended design problems. These are some of the motivations described by Davis [2], who incorporated a heat exchanger design project into a core chemical engineering heat transfer course at the University of Minnesota Duluth. Giving students an opportunity to gain practical, hands-on mechanical skills was in response to feedback from industrial advisors who stated that students were lacking these skills upon entering the work place.

Research on project-based learning methods have found that they result in better performance on assessments of conceptual understanding, improve the ability to solve complex problems, and improve attitudes towards learning [3]. Students are generally also more motivated and demonstrate better communication and teamwork skills.

There are several challenges related to running design projects in a core engineering course. Students in their third semester of engineering typically only have minimal experience with the engineering design process, and therefore it is necessary to be flexible in its application. The large class size, typically 250 to 300 students at Dalhousie, can make it difficult to schedule lab time and to purchase an adequate amount of materials. Further, second-year engineering students already tend to have a relatively high workload, so it is important to make sure that an additional course project will not overwhelm them.

Other challenges are related to the structure of the project. If students are permitted to proceed without any analysis or design planning, the student may not be developing their conceptual thinking and design skills [4]. Failure of a group to achieve a successful prototype may leave a lasting negative impression on the student. Further, some students may struggle with self-directed learning, so the success of the project depends on the level of support and guidance provided by the instructor to help the students learn how to learn on their own.

This paper presents a summary of our experiences with implementing these short design projects over the past three years. The course structure is summarized and the past three design projects are described. The results from these projects are then analyzed from a student experience and pedagogical standpoint. The hope is that this paper will provide useful information for other educators interested in implementing short design projects in their core engineering courses.

\section{COURSE OVERVIEW}

\subsection{Structure and Objectives}

Introductory thermodynamics and fluid mechanics courses are typically taught separately. Since 2011 at Dalhousie, introductory thermodynamics and fluid mechanics have been taught in a single third-semester course to students in all engineering disciplines. Obviously such a course cannot incorporate all of the material that would be covered in separate introductory courses, therefore students in disciplines requiring deeper thermodynamics and/or fluid mechanics knowledge take appropriate additional courses. However, the intention of this first course is to provide students with a basis in both of these important topics. A further objective in teaching this material in an integrated manner is to permit students to see the common first principles shared by these topics. Examples of some of the connections that students typically struggle to make are the link between the first law of thermodynamics and the Bernoulli equation, and the link between the second law of thermodynamics and fluid friction.

The course has three lecture hours, three tutorial hours, and two laboratory hours per week. Lectures are taught to the entire group of students in an auditorium. Tutorials and labs are taught in sections of 50 to 80 students. There are typically six formal laboratory experiments, and one four-week design project. There are typically no labs during the two midterm exam weeks.

Over the last three years, the design projects have involved a build and test component. We have normally divided the class into groups consisting of four to six students. The students are given the project description and asked to reflect on the problem. They then have two work periods (over two weeks) to plan and construct the prototype. Testing is normally done during the third work period. At this point groups normally have a sufficient amount of results to write their report. However, to provide some margin of error, a fourth work period is normally available for additional testing a few days before the report due date.

\subsection{Course Projects}

In developing specific design projects for the thermofluids engineering course at Dalhousie, we have usually relied on discussions with colleagues and brief reviews of the engineering education literature to gather ideas. The last three design projects are described below.

\subsubsection{Ranque-Hilsch Vortex Tube Inserts (Fall 2012).}

A Ranque-Hilsch vortex tube is a mechanical device that produces hot and cold gas streams from a compressed gas stream. These devices are commonly used in applications where a supply of compressed air is readily available and where local cooling is required. The efficiency of these devices is considerably lower than for traditional air conditioning or refrigeration systems. However, their simplicity combined with the fact that they have no moving parts makes them ideal for operation in harsh environments. 
In a typical vortex tube, compressed air enters a chamber which induces the gas to rotate or spin. The chamber usually contains a small metal or plastic insert that directs the fluid and induces the optimal angle of fluid rotation. This insert is commonly referred to as the "Generator" or "Vortex Generator". The ratio of the flowrates of the hot and cold gas streams and their temperature levels can normally be adjusted using a valve on the hot air outlet.

The vortex tube is a relatively simple and affordable way to demonstrate the basics of the first and second laws of thermodynamics. Further, students must apply concepts such as the ideal gas law, as well as mass, energy, and entropy balances in the analysis of such a device [5]. The results can also be used to estimate the coefficient of performance (COP) by comparing the heat output of the cold side to the energy consumed by the compressor [6].

For the project, a commercial vortex tube was purchased from EXAIR ${ }^{\circledR}$ and tested by the students by measuring inlet and outlet pressures and temperatures, and outlet flowrates. The students were responsible to design and build their own generators, and to test and compare the results from their design to the commercial design. Designs were drawn using Solid Edge 3D CAD software and created using a $3 \mathrm{D}$ printer. The test station used to make the measurements is shown in Fig. 1.

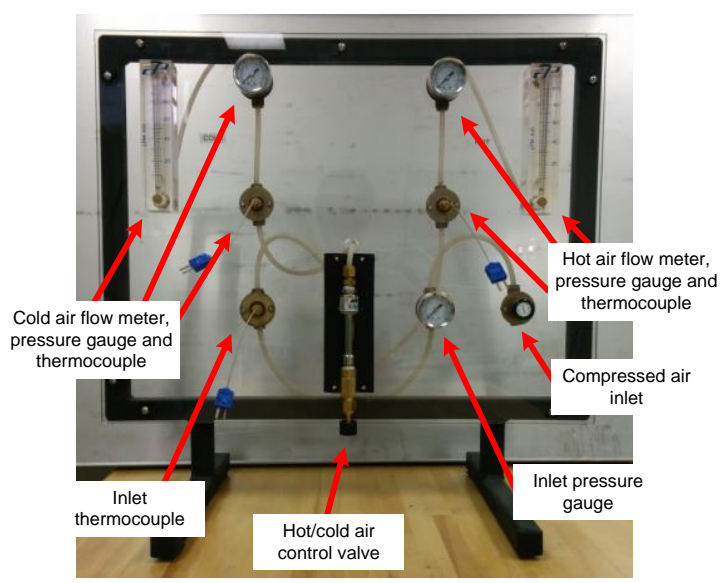

Fig. 1. Vortex tube test station.

2.2.2. Pop-pop Boats (Fall 2013). A pop-pop (also called putt-putt) boat is a toy boat powered by a simple steam engine without any moving parts. The steam engine usually consists of two or more tubes protruding from the rear of the boat. On the other end, the tubes are attached to a reservoir. This reservoir is normally either a separate space or the tubes themselves arranged in a coil. During operation, a heat source (e.g., a candle) supplies heat to the reservoir, causing some of the water to vaporize. The generated steam expands and expels water present in the tubes. This expulsion of water provides forward motion to the boat. Once the expanding steam reaches the cooler section of the tube, it condenses. Eventually enough steam condenses to generate a low pressure in the reservoir, and as a result water flows into the ends of the tubes. This cycle is then repeated as long as heat is supplied to the system. The overall mechanism is similar to that in a pulsejet engine.

Although typically only used as a toy, the pop-pop boat provides an interesting way to demonstrate some key topics in thermofluids, such as the use of steam to generate work, the changing properties of fluids throughout a power cycle, the second law of thermodynamics, and propulsion. Detailed discussions of the operating principles behind the pop-pop boat can be found in many sources [7-9].

To build their pop-pop boat, students were supplied with one 12-inch by 12 -inch sheet of aluminum, one 12 inch by 24-inch sheet of aluminum, one 48-inch length of $1 / 8$ " OD copper tubing, one 48 -inch length of $3 / 16$ " OD copper tubing, five straws, one 1-ounce tube of epoxy, two tealights (candles), one half-pound bag of paraffin wax beads for making candle(s), and candle wick. Additionally, students could use empty pop cans and/or pop bottles. To construct the boats, the students had access to typical hand tools and a drill press.

The performance of the boats was determined by measuring both speed and thrust. The speed was measured by timing how long it took to complete a lap in the project race track (i.e., an 80-inch diameter blow-up pool). The thrust was measured by using a small spring scale. Using the thrust and the average speed, a rough estimate of the power output by the engine was obtained and used to estimate the efficiency of the engine. Students were expected to submit a CAD drawing of their final boat design, along with the completed prototype.

2.2.3. Double Pipe Heat Exchangers (Fall 2014). The heat exchanger project was based on the project described and implemented by Davis [2], which was also used as inspiration for a similar project in the biotechnology program at Dublin City University [10]. Heat exchangers are essential in many process industries. While there a many configurations of heat exchangers depending on the application and the fluids involved, the simplest version of a heat exchanger is the double-pipe heat exchanger. A prototype can be made from copper and PVC (polyvinyl chloride) pipe. A hot fluid (e.g., hot tap water) flows through the copper tubing on the inside of the heat exchanger, while a cold fluid (e.g., cold tap water) flows through the PVC pipe surrounding the copper tubing. As the fluids flow through the exchanger, heat is transferred from the hot to the cold fluid. By measuring the flow rates and change of temperatures for both hot and cold streams, the heat transferred from the hot stream to the cold stream can be determined through an open system, steady-state energy balance. 
To build their double pipe (or shell and coil) heat exchangers, students were supplied with one 1-foot length of 2" PVC pipe, one 6-foot length of 3/16" OD copper tubing, and enough connections and fittings to be able to connect to the test station. Additionally, students had access to PVC pipe glue (primer and cement), Teflon ${ }^{\circledR}$ tape, and some other odds-and-ends in the lab. They also had access to typical hand tools in the lab.

The primary design component of the project was in choosing how to arrange the copper tubing inside the PVC pipe, with the two most popular designs being a coil and a multi-pass tube arrangement. After construction, the heat exchangers were tested and the students applied the steady-state energy balance equation to calculate the rate at which heat was released by the hot fluid and absorbed by the cold fluid, and explain any discrepancy between the two values. Also, comparisons between different designs could be made to determine the best arrangement for the copper tubing.

The test station used to make measurements for the project is shown in Fig. 2. The temperatures were measured at the inlet and outlet for both streams. The flow rates of both the hot and cold streams were also measured. Students were expected to submit a CAD drawing of their final heat exchanger, along with the completed prototype.

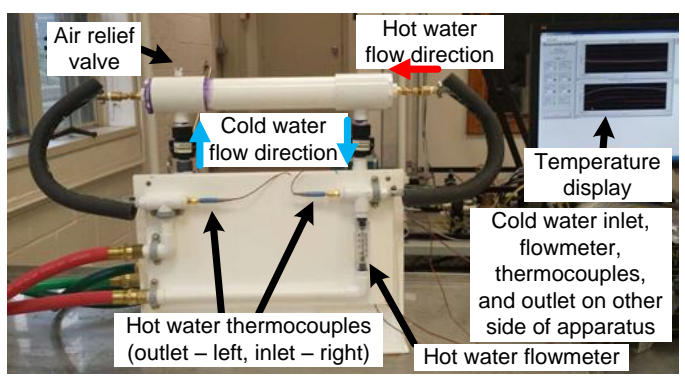

Fig. 2. Heat exchanger test station.

\section{RESULTS AND DISCUSSIONS}

\subsection{Project Quality}

Figure 3 shows the sample vortex tube insert that we showed the students at the beginning of the vortex tube project to give them a basis for their designs. The sample design was purposely inefficient, so that there was significant room for improvement. We also explained to the students that the 3D printer had a certain tolerance, and that approaching this tolerance too closely might lead to structural failure.

Inevitably, some students did print designs that were too complicated to be accurately replicated by the $3 \mathrm{D}$ printer, and ultimately these designs did not work very well. However, many students did synthesize designs that were more efficient than the sample. Examples of two of these designs are shown in Fig. 4. These designs primarily improved upon the sample because they provide space for air to evenly distribute before entering the generator. Other designs investigated the trajectories of the vanes and various gap sizes. There were also various unique designs incorporating a strategically placed hole, but many of these did not work very well due to the resolution of the $3 \mathrm{D}$ printer.

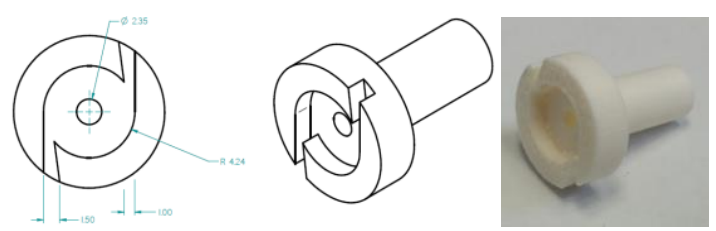

Fig. 3. Sample vortex generator.

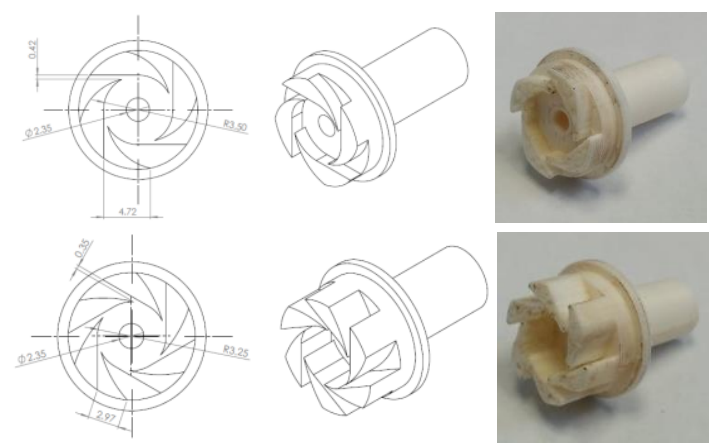

Fig. 4. Examples of student vortex generator designs.

Figure 5 shows one of the two sample pop-pop boats that we showed the students at the beginning of the boat design project. We made it clear to the students that these designs were based on a very brief literature review, and that they were by no means optimized. We encouraged the students to perform their own comprehensive literature review and base their design on logical arguments and some small calculations. We warned them that it would be relatively difficult to build very complex hull designs with the hand tools that were available. Additionally, we warned the students to make sure that their boats would be stable when placed in water.

In the end, most students built relatively simple hull designs and focused their efforts on making candles and efficient boilers. Many groups started by constructing two prototypes and then eliminated one of these when they realized that one performed significantly better than the other. There were many unique single-coil and double-coil copper tube boiler designs with varying sizes. Additionally, some groups constructed boiler reservoirs using pop cans. Eventually most groups realized that the performance of their boat could be improved by maximizing the amount of heat transferred to the boiler. Therefore most groups focused their final design iterations on maximizing the surface area of the boiler and changing the candle size and location. Two example boat designs are shown in Fig. 6. 


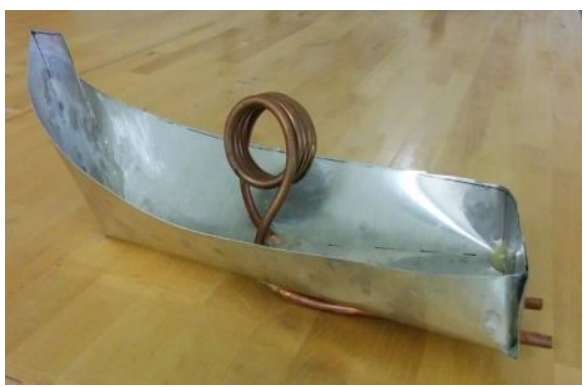

Fig. 5. Sample pop-pop boat.
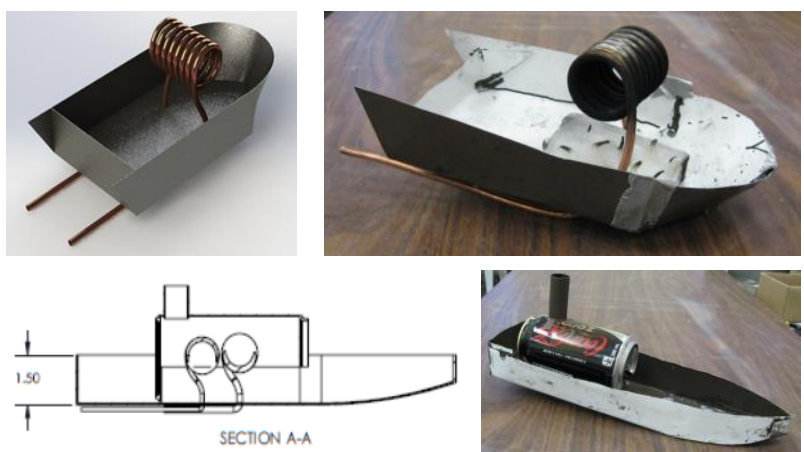

Fig. 6. Examples of two student pop-pop boat designs: CAD drawing (left) and completed prototype (right).

Similar to the sample vortex tube insert and pop-pop boat, the sample heat exchanger, shown in Fig. 7, did not use the most creative or best design with regards to the use of copper tubing. The copper tubing ran straightthrough the PVC pipe, minimizing the available heat transfer area. The use of clear PVC pipe, while too expensive to use for all of the projects, effectively displayed the inner workings of the heat exchanger.

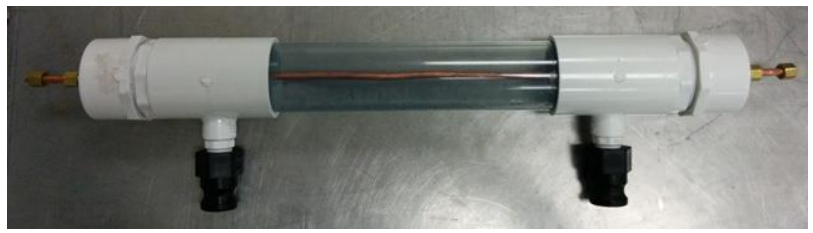

Fig. 7. Sample heat exchanger.

The students were encouraged to develop a more creative arrangement of the copper tubing within the PVC pipe, and some examples are shown in Fig. 8. We recommended that they included the full length of copper tubing inside the PVC pipe to maximize heat transfer area. The secondary goal was to arrange the tubing such that it created turbulence in the water flowing on the outside of the copper tubing. The simplest designs were the standard coil (Fig. 8a) or a multi-pass arrangement (Fig. 8b). More creative designs incorporated both coil and multi-pass aspects (Fig. 8c and d) or improved on the standard coil by using changing diameters to promote turbulence (Fig. 8e). Fig. 8f shows an example of a group's completed CAD drawing.
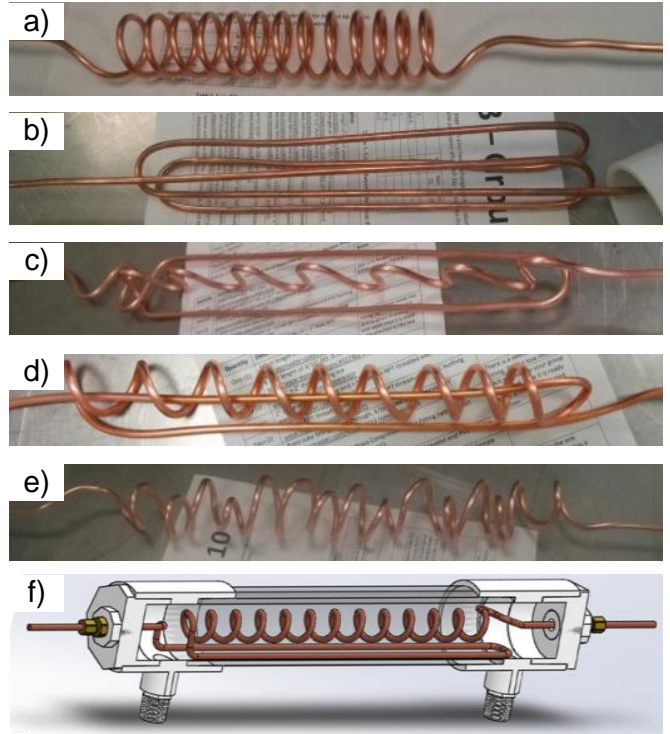

Fig. 8. Examples of student heat exchanger designs: a) standard coil design, b) multi-pass design, c) serpentine design, d) combined coil and multi-pass design, e) coil with changing diameter, f) CAD drawing.

\subsection{Analysis and Student Feedback}

In all three years, the students displayed a high level of enthusiasm toward the design project. This was especially evident for the students who either had previous experience with build projects or who had a keen interest in developing these skills. The projects gave students who already had strong hands-on skills an opportunity to excel and even teach their less experienced classmates.

Although a few students brought these skills to the project, it was also evident that most students had a minimal amount of hands-on practical skills at this stage in their education. Specific skills obtained from the vortex tube project (Fall 2012) include learning how to use 3D printers, how to fasten compression fittings, and how to read rotameters and pressure gauges. Specific skills obtained from the pop-pop boat project (Fall 2013) include learning how to work with copper tubing and sheet metal and how to apply epoxy. Specific skills obtained from the heat exchanger project (Fall 2014) include learning how to fasten compression fittings, connect PVC pipe, apply epoxy and Teflon ${ }^{\circledR}$ tape, and read rotameters. Additionally all three of these projects exposed the students to some basic hand tools. Though these skills seem trivial to experienced engineers and technicians, most early year engineering students have never been exposed to them before. Having even minimal experience will improve confidence and put them in a better position to be productive as junior engineers.

Whether the students' understanding of the core concepts involved in the project improved as a result of the project is difficult to measure. Clearly, connections 
between the project and the course material must be made when writing the report, but it cannot be guaranteed that all students will benefit equally from this aspect of the project. It is therefore the instructor's responsibility to help make these connections by incorporating the project into lectures and giving clear instructions about how to apply calculations from the course to the project.

In general, students gave positive comments while working on the projects. Only a few formal comments were given in the end of year course evaluations. In 2014, one student mentioned that they thoroughly enjoyed the project. Another commented that the concepts needed to analyze the heat exchanger design were beyond the scope of the course. Although this may be true, it can be argued that the foundational knowledge gained from the project will be helpful to the students who will study heat exchangers in greater detail in future heat transfer courses. Further, it is relatively easy to challenge such an argument because even practicing engineers rarely know every detail about all related topics when confronted with a new design problem.

Another negative comment was that there was not enough room for creative design in the heat exchanger project. This highlights a challenge in developing short design projects: they must be open-ended enough to encourage student creativity and effectively apply design principles, yet constrained enough so that it is still possible to come up with a working prototype within a short period of time. From our experience, short design projects such as this must be relatively well constrained with students only focusing on specific aspects of the design. Leaving the project too open-ended can lead to frustration and cause some students to turn too much focus away from the core course material. Keeping the project constrained gives the students an achievable goal and allows them to fully benefit from the experience.

\section{CONCLUSIONS}

In general, incorporating short design projects into the introductory thermofluids course at Dalhousie has been a positive experience. The major challenge is managing the additional time and resources required to develop and run the project. Project development, part sourcing, and prototype building and testing should be done during a prior semester to avoid last minute stresses during the semester.

If much of the project can be prepared ahead of time, undertaking short design projects can be rewarding for both the students and the instructors. The benefits include reaching out to students whose learning styles do not correspond well to traditional teaching methods, giving the students exposure to basic hand tools and construction materials, and developing their design and teamwork skills.

\section{Acknowledgements}

Financial support from Dalhousie's Faculty of Engineering and the Shell Experiential Learning Fund (SELF) is gratefully acknowledged. Helpful advice from Dr. Michael Pegg and Dr. Clifton Johnston is also gratefully acknowledged. We would also like to thank the students of ENGI 2102 from fall 2012, 2013 and 2014 for their participation in the design projects and useful feedback.

\section{References}

[1] Richard M. Felder and Linda K. Silverman, "Learning and Teaching Styles in Engineering Education," Engr. Education, vol. 78, no. 7, pp. 674-681, 1988.

[2] Richard A. Davis, "A Project to Design and Build Compact Heat Exchangers," Chem. Eng. Educ., vol. 39, no. 1, pp. 3841, Winter 2005.

[3] Michael J. Prince and Richard M. Felder, "Inductive Teaching and Learning Methods: Definitions, Comparisons, and Research Bases," J. Engr. Education, vol. 95, no. 2, pp. 123-138, 2006.

[4] Andrew Samuel, Make and Test Projects in Engineering Design. London: Springer-Verlag, 2006, 47 pp. \{ISBN $1852339152\}$

[5] Edorta Carrascal and José M. Sala, "Mass, energy, entropy and exergy rate balance in a Ranque-Hilsch vortex tube," Journal of Technology and Science Education, vol. 3, no. 3, pp. 122-131, 2013.

[6] Don Van, Joel Ingram, Kenneth Mayo, and Kian Jost, "Process Analyzing of the Vortex Tube and The Teaching and Learning of Energy Efficiency and Sustainability," in ASEE American Society of Engineering Education Southeast Section Conf., (Cookeville, TN; 10-13 March 2013), 2013.

[7] Jeff Bindon, "The Secret Workings of a Transparent PopPop Engine," Model Engineer, vol. 39, no. 1, pp. 38-41, Winter 2005.

[8] V Sharadha and Jaywant H Arakeri, "Propulsion of the Putt-Putt Boat - I," Resonance, vol. 9, no. 6, pp. 66-73, 2004.

[9] V Sharadha, "Propulsion of the Putt-Putt Boat - 2," Resonance, vol. 9, no. 7, pp. 64-69, 2004.

[10] Brian Freeland, John M. Tobin, and Greg Foley, "A Project and Competition to Design and Build a Simple Heat Exchanger," in Proc. of the International Symposium for Engineering Education, ISEE-07, Dermot Brabazon and Abdul-Ghani Olabi (eds.) (Dublin, Ireland; 2007), pp. 201206, 2007. 